\title{
The OSIRIS-REx Visible and InfraRed Spectrometer (OVIRS): Spectral Maps of the Asteroid Bennu
}

\author{
D.C. Reuter ${ }^{1}$ A.A. Simon ${ }^{1}$ - J. Hair ${ }^{1}$ - A. Lunsford ${ }^{2}$ - S. Manthripragada M V. Bly $^{1}$. \\ B. Bos ${ }^{1}$ - C. Brambora ${ }^{1}$ - E. Caldwell ${ }^{1}$. G. Casto ${ }^{1}$ - Z. Dolch ${ }^{1}$ - P. Finneran ${ }^{3}$. \\ D. Jennings ${ }^{1}$ - M. Jhabvala ${ }^{1}$. E. Matson ${ }^{1} \cdot$ M. McLelland ${ }^{4}$ - W. Roher ${ }^{1} \cdot$ T. Sullivan ${ }^{5}$.

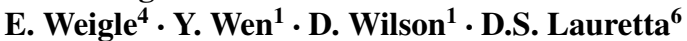

Received: 27 February 2017 / Accepted: 2 February 2018 / Published online: 5 March 2018

(C) The Author(s) 2018. This article is published with open access at Springerlink.com

\begin{abstract}
The OSIRIS-REx Visible and Infrared Spectrometer (OVIRS) is a point spectrometer covering the spectral range of 0.4 to 4.3 microns $\left(25,000-2300 \mathrm{~cm}^{-1}\right)$. Its primary purpose is to map the surface composition of the asteroid Bennu, the target asteroid of the OSIRIS-REx asteroid sample return mission. The information it returns will help guide the selection of the sample site. It will also provide global context for the sample and high spatial resolution spectra that can be related to spatially unresolved terrestrial observations of asteroids. It is a compact, low-mass $(17.8 \mathrm{~kg}$ ), power efficient ( $8.8 \mathrm{~W}$ average), and robust instrument with the sensitivity needed to detect a 5\% spectral absorption feature on a very dark surface (3\% reflectance) in the inner solar system (0.89-1.35 AU). It, in combination with the other instruments on the OSIRIS-REx Mission, will provide an unprecedented view of an asteroid's surface.
\end{abstract}

Keywords OSIRIS-REx · Bennu $\cdot$ Asteroid $\cdot$ Composition $\cdot$ Visible and infrared spectrometer $\cdot$ Spectral maps

OSIRIS-REx

Edited by Dante Lauretta and Christopher T. Russell

$\triangle$ D.C. Reuter

dennis.c.reuter@nasa.gov

1 NASA/GSFC, Greenbelt, MD, USA

2 Catholic University of America, Washington, DC, USA

3 Jackson and Tull Inc., Beltsville, MD, USA

4 SwRI, San Antonio, TX, USA

5 Muniz Engineering, Seabrook, MD, USA

6 University of Arizona, Lunar and Planetary Laboratory, Tucson, AZ, USA 


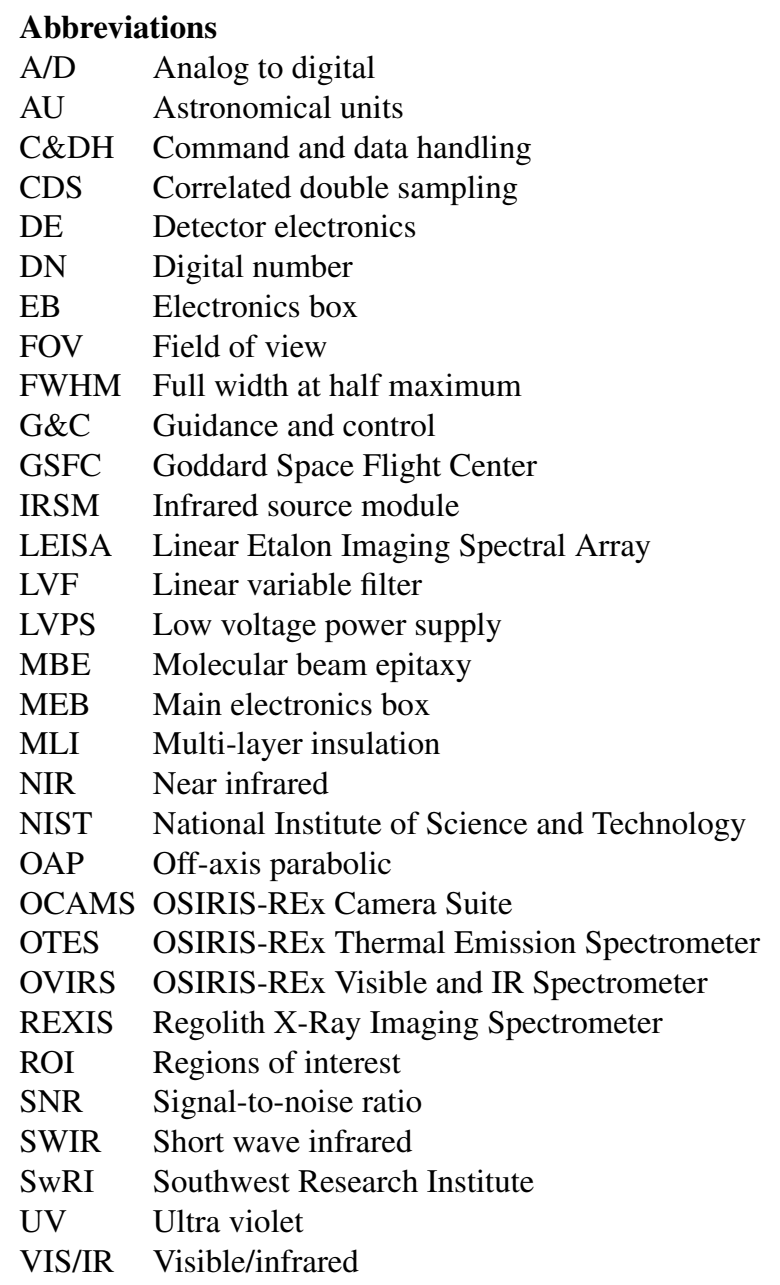

\section{Introduction}

The OSIRIS-REx Visible and IR Spectrometer (OVIRS) is a point spectrometer with a 4mrad diameter circular field of view (FOV) that provides spectra over the wavelength range of 0.4-4.3 $\mu \mathrm{m}\left(25,000-2300 \mathrm{~cm}^{-1}\right)$. It employs wedged filters (also called linear variable filters) to provide the spectrum. A wedged filter is a two-dimensional spectral filter in which the wavelength of transmitted light varies with position along one of the spatial dimensions. The OVIRS design is based on the New Horizons LEISA instrument design (Reuter et al. 2008), but with simplified optics and an extended wavelength range.

The OVIRS spectral resolution requirements were defined in terms of resolving power, $R$ $(=\lambda / \Delta \lambda$ where $\Delta \lambda$ is the full width at half maximum (FWHM) of the spectral distribution function), and are $R \geq 125$ in the 0.4 - to $1.1-\mu \mathrm{m}$ spectral range; $R \geq 150$ in the 1.1 - to $2.0-\mu \mathrm{m}$ spectral range; and $R \geq 200$ in the 2.0 - to $4.3-\mu \mathrm{m}$ spectral range. In addition, the spectral range from 2.9 to $3.6 \mu \mathrm{m}$ was required to have $R \geq 350(\Delta \lambda<10 \mathrm{~nm}$ ) to resolve key organic spectral features, such as those that have recently been observed on the asteroids 
24 Themis (Campins et al. 2010; Rivkin and Emery 2010), Ceres (De Sanctis et al. 2017) and (65) Cybele (Licandro et al. 2011). As is discussed in the sections that follow, OVIRS meets these requirements with margin on the lower limit.

The OVIRS spectral range and resolving powers were optimized to provide surface maps of mineralogical and molecular components including carbonates, silicates, sulfates, oxides, adsorbed water, and a wide range of organic species, as well as the products of space weathering and the visible contribution to the Yarkovsky effect (e.g., Vokrouhlicky et al. 2000; Gaffey 2010). It is a point spectrometer, so the full spectrum of an area on the target surface corresponding to the FOV is obtained in a single frame. OVIRS operates in a scanning mode, in which the cross-track rotational motion of the asteroid is combined with rotations of the spacecraft that scan the OVIRS boresight along-track to sample a region of interest and build up a global data set. For example, global data are collected by scanning the OVIRS FOV in a N-S direction by moving the spacecraft, while Bennu rotates in an E-W direction. The spacecraft scans at a rate that allows the N-S scans to be accomplished in a time sufficiently less than the time it takes for the E-W rotation of the asteroid to move an OVIRS FOV that the there are no gaps in the coverage. In the expected operational scenario, OVIRS will provide full-disk asteroid spectral data, global spectral maps (at 20-m resolution or better), and spectra of the sample site (at 0.08 - to 2-m resolution). The full disk spectra will be obtained at a phase angle between 2 and 15 degrees, and global spectral maps will be obtained at solar phase angles of approximately 5,30,45, \pm 90 and \pm 135 degrees. The instrument provides at least two spectral samples per spectral resolution element (spectral double sampling) taking full advantage of the spectral resolution. OVIRS spectra will be used to identify volatileand organic-rich regions, if they exist. These data will be used in concert with data from the other OSIRIS-REx instruments (OCAMS (OSIRIS-REx Camera Suite, a 3-camera imaging system), OLA (OSIRIS-REx Laser Altimeter), OTES (OSIRIS-REx Thermal Emission Spectrometer) and REXIS (Regolith X-Ray Imaging Spectrometer)) to guide sample-site selection and provide an unprecedented global inventory of the composition and regolith structure of the asteroid's surface.

OVIRS uses an off-axis parabolic (OAP) mirror to image the surface of the asteroid onto a field stop. The field stop selects a 4-milliradian angular region of the image. The light from this 4-milliradian area passes to a second OAP that re-collimates it and illuminates the Focal Plane Assembly. Because the beam speed is low $(\sim \mathrm{f} / 50)$ this assembly, consisting of the array with the filter mounted in close proximity to it, is effectively at a pupil. Each detector element of the array "sees" the same spatial region of the asteroid but, as described in Sect. 3.1, different columns of the array "see" it at different wavelengths. Nearly $96 \%$ of the instrument's response to light is contained within the 12.57-microsteradian solid angle corresponding to this 4-milliradian cylinder. Within the cylinder, the response is flat to a radius of 1.8 milliradian, dropping linearly to the response at a radius of 2 milliradian. The details of the enclosed energy response outside of 4 milliradian are given in Sect. 5.1. The complete spectrum of the sensed spot is obtained in a single measurement. This is somewhat different than the case for some wedged filter spectral imagers, such as LEISA on New Horizons, where the spectrum of a given point is built up over several frames (e.g., Reuter et al. 2008).

The detector array is thermally coupled to the cold, second stage of a two-stage passive radiator to obtain focal plane temperatures $<110 \mathrm{~K}$. This reduces the dark current to $<200 \mathrm{e}^{-} / \mathrm{s}$. so that dark current noise is never the dominant noise source with more than a factor of two margin (see Sect. 4). The camera enclosure shields its contents from radiation and contaminants and mounts to a flange attached to the bottom of the OSIRIS-REx spacecraft nadir deck. A cold baffle in the optical path limits the thermal background signal from 
the instrument enclosure. In addition, the first stage of the two-stage radiator is attached to the optics enclosure and keeps its temperature less than $160 \mathrm{~K}$, further reducing thermal background noise.

OVIRS was calibrated prior to launch (see Sects. 4 and 5), and the calibration will be checked throughout the mission. Ground spectral calibration was accomplished using gratings to provide tunable narrow-band radiometric sources. In addition, lasers and narrowband atomic lamp sources provided additional information. Radiometric and relative response calibrations were performed using NIST (National Institutes of Science and Technology) traceable calibrated blackbodies and integrating spheres. The quality of the point spread function was assessed using collimated point and extended sources. The boresight pointing was measured with respect to optical alignment cubes on the spectrometer.

In-flight radiometric calibration will rely on three methods: an onboard array of miniature blackbody sources ( $T \sim 700 \mathrm{~K}$ for IR wavelengths) placed at the OVIRS field-stop and tungsten filament sources (spectral coverage from $\sim 0.6$ to $4 \mu \mathrm{m}$ ) located behind the secondary mirror (all calibrated prior to launch), in-flight observations of the Earth and the Moon through the main instrument aperture, and solar radiance calibrations using a secondary input to the optical system. The internal sources allow regular monitoring of the instrument's relative calibration over wavelengths from 0.6 to $4 \mu \mathrm{m}$, while the external sources provide periodic checks on the absolute spectral and radiance calibration over all wavelengths. The terrestrial and lunar calibrations will occur on the OSIRIS-REx flyby of Earth in 2017 (see Lauretta et al. 2017, this issue, for details). The on-board solar radiance calibrations will be carried out occasionally by using the spacecraft control system to point the solar calibration port at the Sun. The combination of these methods will provide redundant radiometric calibration. OVIRS will provide spectral data with at least $5 \%$ radiometric accuracy and no worse than $2 \%$ pixel-to-pixel precision. Because wedged filters are very stable, the spectral calibration is not expected to change in flight; however, the Earth and Lunar observations will also provide spectral calibrations. Spectral calibration is expected to be accurate to 0.25 of a spectral element halfwidth or better. The dark current and background flux will be measured using dark sky observations. For most observational types the OVIRS FOV will be more than 12 milliradian off of Bennu for more than 30 seconds at the beginning and/or end of each scan. Measurements made during these times that do not have the internal sources on constitute the dark sky observations for these types. For observational types that keep the OVIRS FOV on Bennu for the entire time, dark sky measurements will be taken with the FOV at least 12 milliradians off of Bennu within an hour of the beginning and end of the observation.

\section{OVIRS Science Overview}

The full scientific rationale for the OSIRIS-REx mission to the asteroid Bennu is given in detail in another paper in this volume (Lauretta et al. 2017). The primary goal of the mission is to return a pristine sample from Bennu, a primitive carbonaceous asteroid. In essence, Bennu is a fossil along the path of planetary evolution, and the analysis of the returned sample will help us understand the processes that occurred in the early Solar System and possibly even give clues to the development of life on Earth (Lauretta et al. 2015). This makes the Mission both scientifically exciting and publicly appealing. It will provide groundbreaking insights into Solar System origins and ongoing processes in the solar system.

OVIRS will play a valuable role in this exploration, connecting ground-based observations to local surface context and to the returned sample. It directly addresses four of the 
Fig. 1 (top) Spectrum of main-belt asteroid Themis showing significant spectral features in the 3-3.5 micron spectral region, characteristic of organic $\mathrm{C}-\mathrm{H}$ stretching bands. (bottom) The Themis spectrum convolved to OVIRS resolution; markers indicate the expected noise amplitude. The spectra were modeled for two different surface temperatures, showing increased thermal emission $(330 \mathrm{~K})$ filling in reflectance spectra in this region. Bennu's surface may reach up to $400 \mathrm{~K}$ in some regions
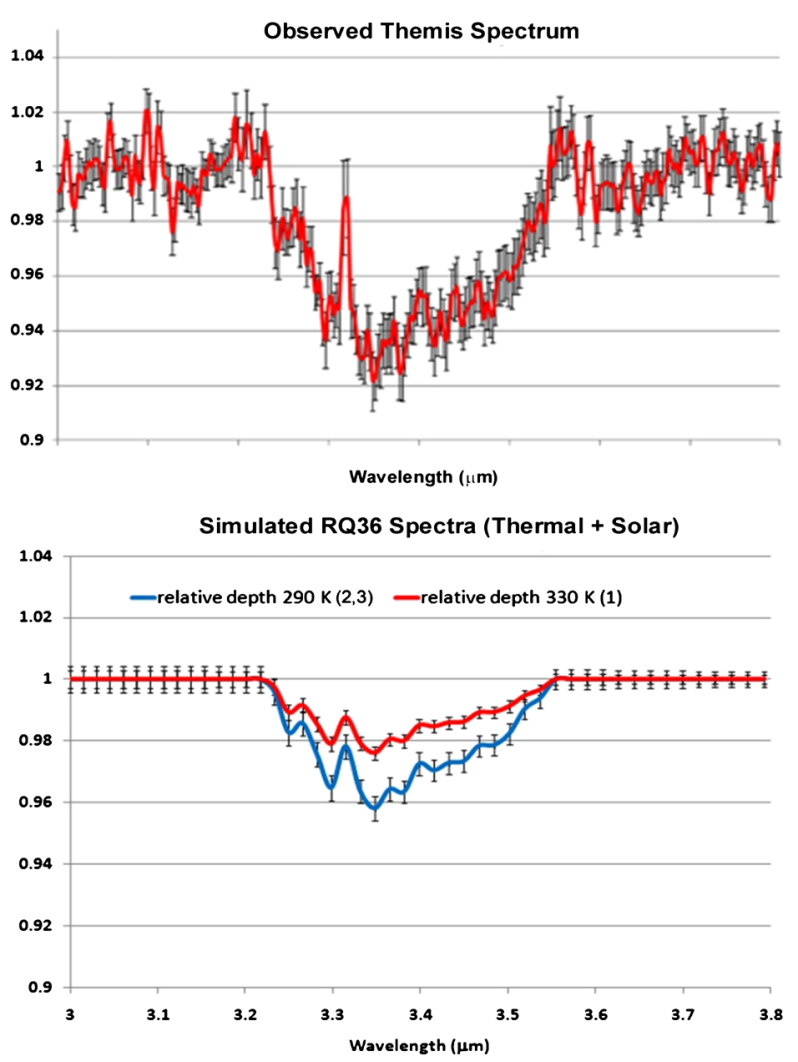

fifteen Level 1 requirements and contributes to three more. Its spectral range, spectral resolution, and noise performance were driven by the need to identify spectral features that correspond to compositional information. These include features associated with adsorbed water, phyllosilicates, carbonates, sulfates, silicates, oxides, and hydrocarbons. Global maps identifying these spectral features and their absorption strengths will be used for assessing which sample sites have the highest science return. These global maps will be obtained at a spatial resolution of $20 \mathrm{~m}$ at seven solar phase angles $\left(5^{\circ},-30^{\circ}, 45^{\circ}, \pm 90^{\circ}\right.$ and $\left.\pm 135^{\circ}\right)$, which will allow photometric correction of the spectra taken at any angle to a reference angle (nominally, $30^{\circ}$ ). The photometrically corrected global spectral maps will be processed using compositional spectral indicators to produce global maps of mineral and chemical composition. These, in turn, will be correlated to a geometric and topographical shape model to allow investigation of possible surface processes, including impacts, and the effects of space weathering. A global bond albedo map will also be produced from these observations.

Once two possible sample sites are chosen, higher spatial resolution OVIRS spectral maps ( 2 meter resolution) of the candidate sample sites will provide detailed context information for the returned sample. They will also provide additional science value information which may be used in choosing the first sample site. For example, the top panel of Fig. 1 shows a spectrum of the asteroid Themis in the wavelength region corresponding to the $\mathrm{C}-\mathrm{H}$ stretch modes associated with organic species (Campins et al. 2010; Rivkin and Emery 2010). Areas where spectral features such as these were observed would be very strong candidates for sample site selection. 
In addition to helping guide the sample site selection process, the OVIRS spectra will provide a link between ground-based telescopic measurements and the more detailed in situ measurements. Also, although the silicate mineralogy of asteroids can be inferred by spectral matching between asteroids and meteorites (e.g., Hiroi et al. 2001), the detailed mineralogy of most asteroids is still uncertain. This is because telescopic measurements and laboratory measurements study inherently different samples. The OVIRS spectra, in conjunction with the laboratory measurements of the returned sample, will provide a link between spectra, which sample the top few microns of an object's surface, and the subsurface composition that will be revealed by the analysis of the returned sample.

Table 1 summarizes the science objectives that determined the OVIRS design, the measurement strategies that address these objectives, and the derived instrument performance requirements. The performance requirements were determined by the need to address the Level 1 objectives that OVIRS was capable of fulfilling by itself.

\section{Opto-Mechanical Design}

Figure 2 shows a model of the OVIRS Optics Box exterior, while Fig. 3 shows a model of the interior of the box and a ray-trace diagram. The major elements are labeled in the models. The Optics Box is mounted to the spacecraft by thermally isolating titanium flexures. Cooled by the first stage of a two-stage radiator, the in-flight temperature of the Optics Box is predicted to range from about $120 \mathrm{~K}$ in cruise to about $150 \mathrm{~K}$ during some asteroid operations. The higher temperature at the asteroid is primarily due to radiative heating of the Optics Box by Bennu, although there is some solar heating as well. The placement of the radiator surfaces and the spacecraft attitudes used during operations are designed taking these effects into account. The low temperature of the Optics Box reduces the conductive and radiative thermal load on the focal plane. It also limits the background signal at the long-wavelength end of OVIRS. The second stage of the radiator was predicted to cool the OVIRS detector to about $90 \mathrm{~K}$ during cruise and to keep it less than $105 \mathrm{~K}$ during asteroid operations. To date in flight (through 2016) the decontamination heaters have been on most of the time. There has only been one 82-hour period when the Optics Box and the detector were allowed to cool, during which the detector reached $106 \mathrm{~K}$ and the optics box reached $155 \mathrm{~K}$. Based on this performance, it was predicted that the detector will have reached a steady-state temperature $<98 \mathrm{~K}$ during cruise and that the maximum temperature during observations at Bennu will be $<110 \mathrm{~K}$. Even at $110 \mathrm{~K}$, the noise contribution of the $<20 \mathrm{e}^{-} / \mathrm{s}$. dark current (idark) for the planned observation times is still much less than the read noise, so the predicted signal-to-noise ratios are not significantly affected (see Sect. 4). Figure 4 shows a picture of the Optics Box with Multi-Layer Insulation (MLI) attached. The instrument parameters for OVIRS are summarized in Table 2.

As may be seen in Fig. 3, the first mirror, which has an 80-mm clear aperture and a focal length of $350 \mathrm{~mm}$, images the incoming scene, either from the primary aperture or the solar calibrator aperture, onto the field stop. The beam at the field stop is $\mathrm{f} / 4.4$. The field stop is a 1.4-mm circular aperture which allows light from a 4-milliradian angle to pass to the secondary mirror. The secondary mirror has an aperture of $20 \mathrm{~mm}$ and a focal length of 88 $\mathrm{mm}$, so that the $\mathrm{f} / 4.4$ beam coming from the field stop fills the aperture of the secondary mirror. Since the field stop is in the focal plane of the secondary mirror, the light leaving it is collimated. This light travels to the focal plane, which is about $150 \mathrm{~mm}$ from the field stop. The angular spread of the light reaching the focal plane is only about \pm 7 milliradian, and the beam overfills the optically active area used on the array such that the uniformity of 


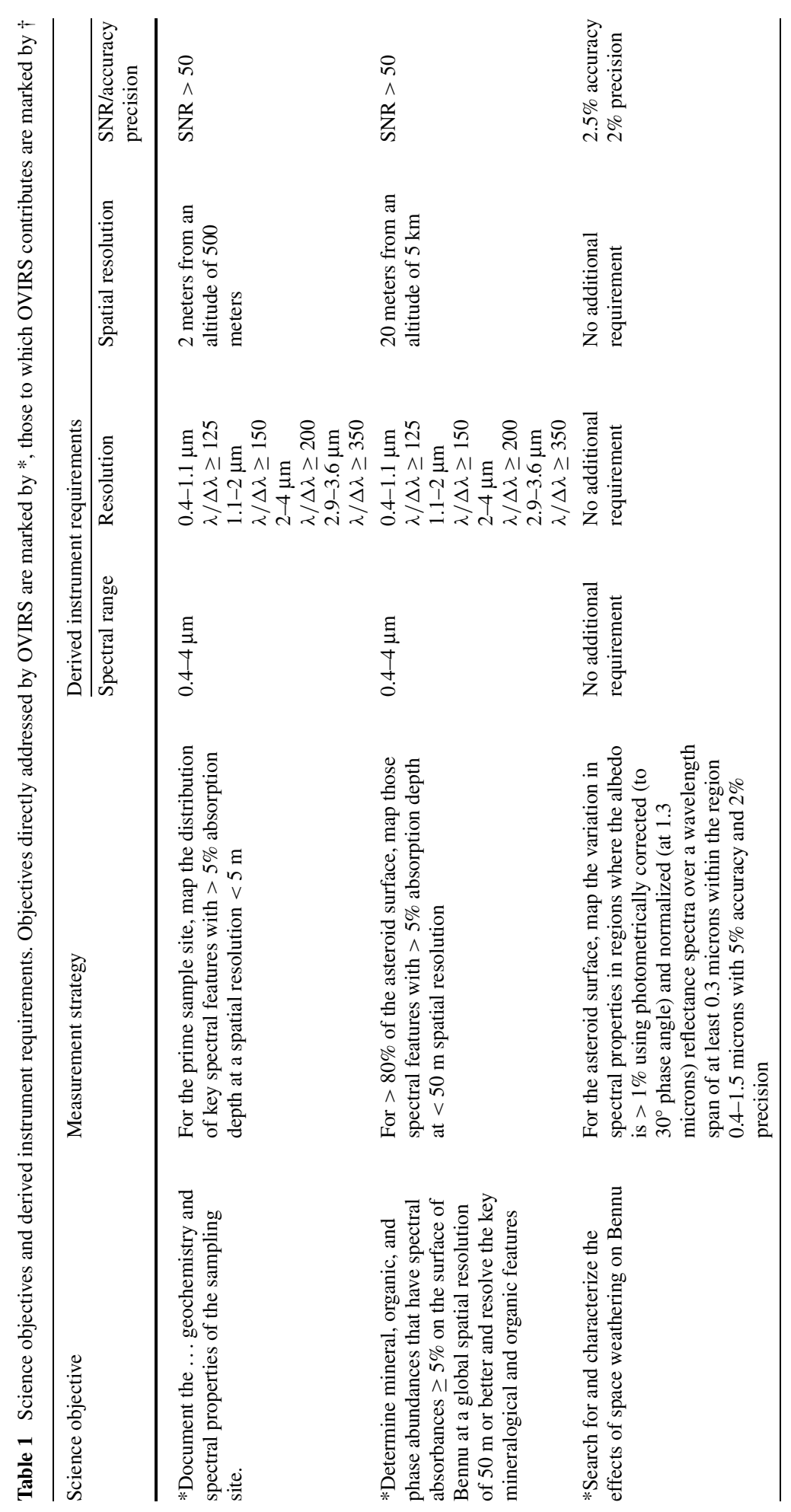




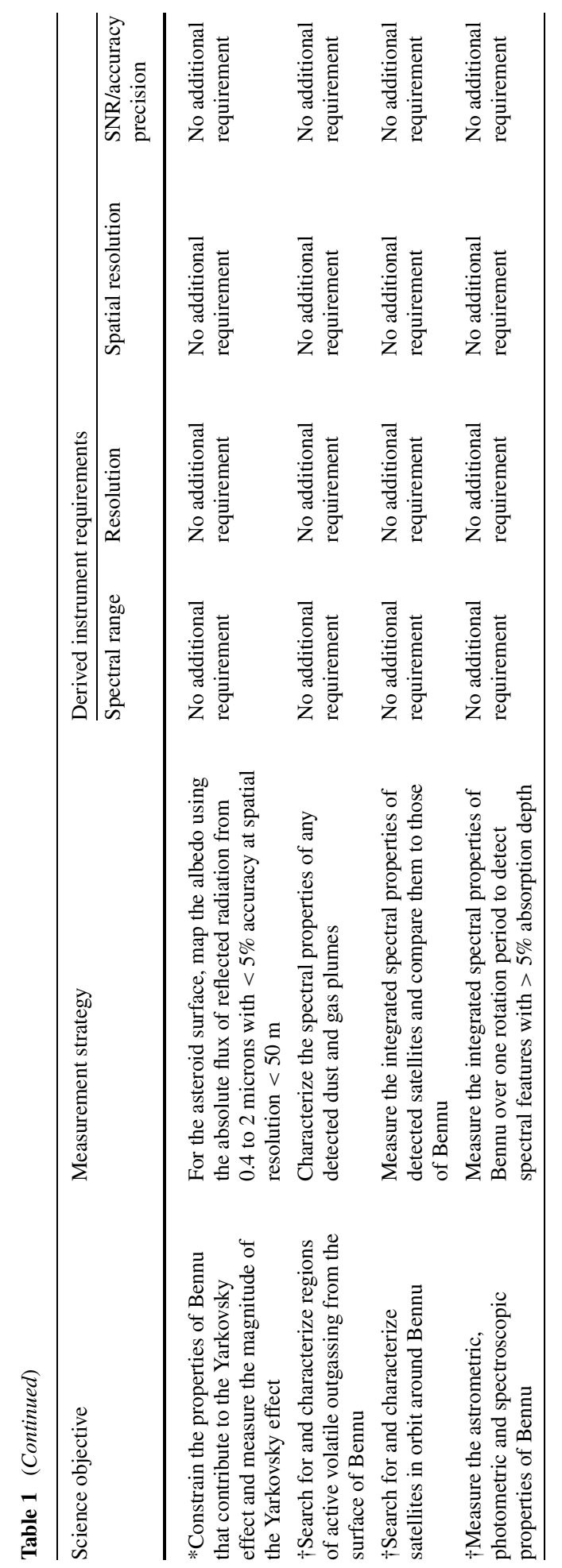




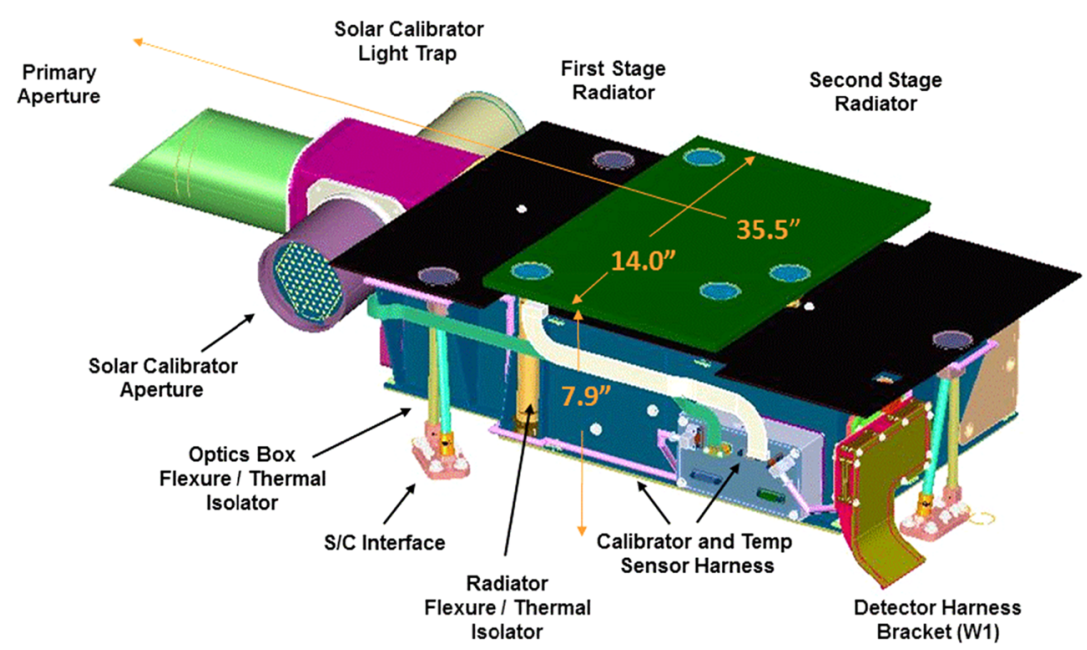

Fig. 2 OVIRS model showing the names and placements of the external components

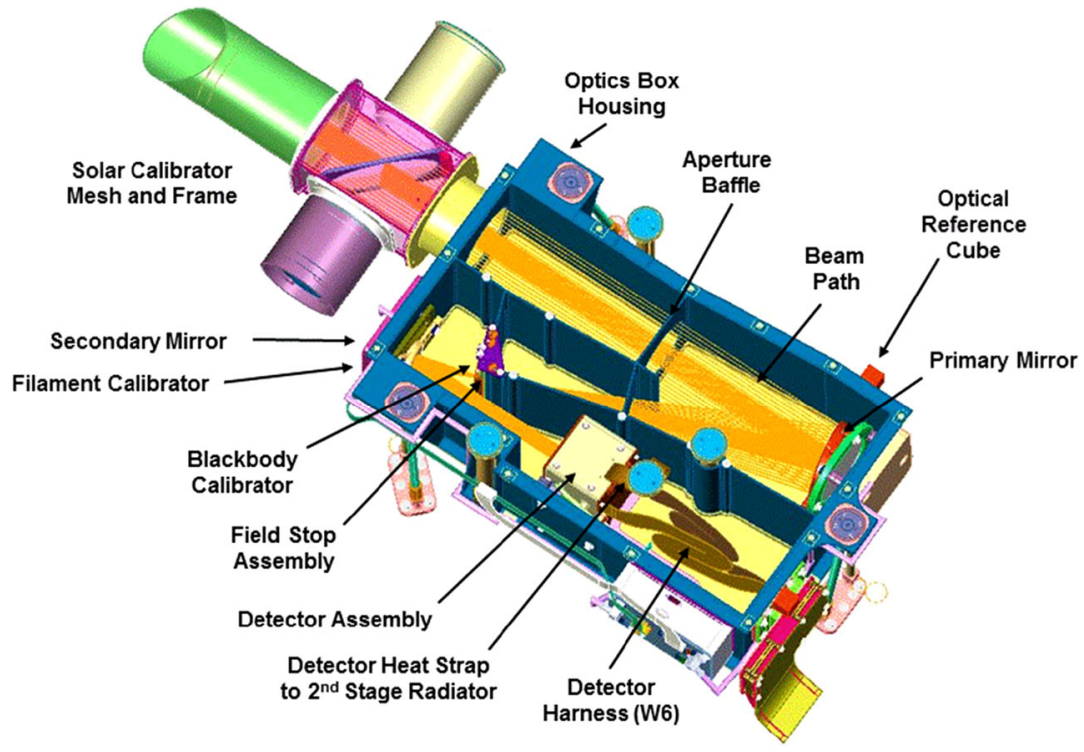

Fig. 3 Model showing the internal structure and components of the Optics Box. All internal surfaces are treated with a nonreflective coating

the field is greater than 0.992. (That is, each pixel in the array "sees" the same area on the ground with more than $99 \%$ overlap.)

There are two electronically activated calibration sources that can illuminate the focal plane. The first of these is a ring of miniaturized blackbody sources that are placed behind the field stop within a few mm of the focal plane of the secondary mirror. These blackbodies, which can reach a temperature of about $700 \mathrm{~K}$, provide collimated IR radiance to the focal plane covering the wavelength range from the $4.3-\mu \mathrm{m}$ limit allowed by the focal plane filters 
Fig. 4 Picture of OVIRS Optics Box showing input and solar calibration apertures. The Box is wrapped in Multi-Layer Insulation (MLI) for thermal isolation

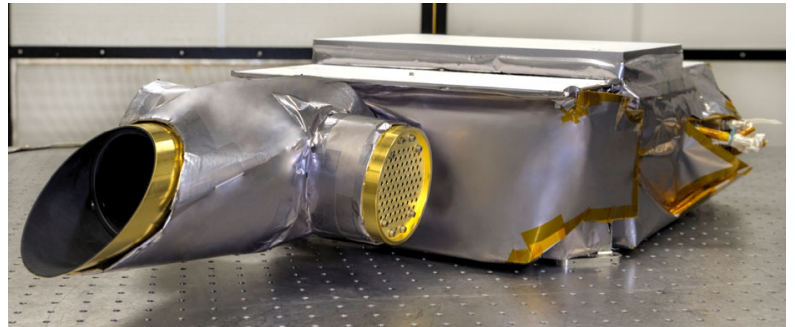

Table 2 OVIRS instrument parameters

Mass: $17.7 \mathrm{~kg}$

Power: 8.8 watt (average), $13.5 \mathrm{~W}$ (peak)

Frame rate: $0.1-25 \mathrm{~Hz}$

Optics Box

Mass: $12.2 \mathrm{~kg}$

Temperature: $<160 \mathrm{~K}$

Telescope aperture: $80 \mathrm{~mm}$

FOV: 4-milliradian circle

Focal plane: $1024 \times 1024$ element H1RG HgCdTe array (only the central $524 \times 524$ region is used)

Pixel size: $18 \mu \mathrm{m} \times 18 \mu \mathrm{m}$

Focal plane temperature: $<110 \mathrm{~K}$

Filter segment 1a $(0.396-0.667 \mu \mathrm{m})$ resolving power $(\lambda / \Delta \lambda) \geq 139$

Filter segment $1 \mathrm{~b}(0.653-1.100 \mu \mathrm{m})$ resolving power $(\lambda / \Delta \lambda) \geq 136$

Filter segment $2(1.079-1.818 \mu \mathrm{m})$ resolving power $(\lambda / \Delta \lambda) \geq 185$

Filter segment $3(1.783-3.004 \mu \mathrm{m})$ resolving power $(\lambda / \Delta \lambda) \geq 242$

Filter segment $4(2.878-4.333 \mu \mathrm{m})$ resolving power $(\lambda / \Delta \lambda) \geq 372$

Main Electronics Box (MEB)

Mass: $4.58 \mathrm{~kg}$

Operational temperature: $273-313 \mathrm{~K}$

Fully block redundant

Three circuit boards

Focal plane electronics (FPE): Controls and reads the focal plane

Command and data handler $(\mathrm{C} \& D H)$ : Command and data interfaces with the FPE and the spacecraft

Low voltage power supply (LVPS): Supplies power to the other boards and to the calibration sources

Electrical harnesses: $0.9 \mathrm{~kg}$

to about $2.5 \mu \mathrm{m}$. There are 32 total miniaturized blackbodies, which are divided into two sets of 16. Each set is powered by one side of the redundant electronics. The second calibration source is a set of four small filament lightbulbs which, similar to the blackbodies, are divided into two redundant pairs of two bulbs each. These bulbs are contained within an enclosure that is mounted to the outside of the Optics Box, behind the secondary mirror. A small aperture in the Optics Box wall allows light from the filament source to directly illuminate the focal plane from a distance of about $180 \mathrm{~mm}$. This source provides usable signal from about $0.6 \mu \mathrm{m}$ to $4.3 \mu \mathrm{m}$. The blackbody and filament calibrators were radiometrically calibrated with NIST traceable sources at a number of power settings during thermal vacuum testing (see Sects. 4 and 5 below). 

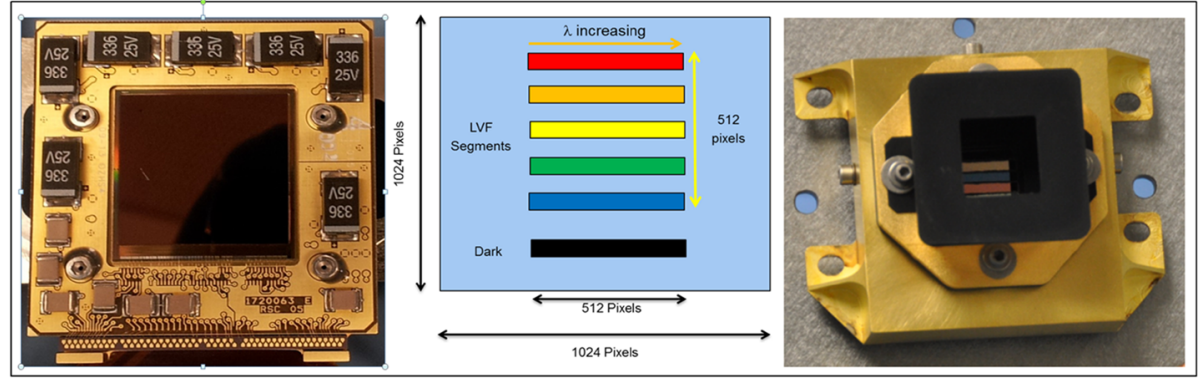

Fig. 5 (left) The Teledyne H1RG $1024 \times 1024$ pixel array with filter capacitors and resisters. (center) Schematic showing how the filter segments are laid out over the array. The black area is a part of the array that is completely covered by the filter holder and is read out to get a measure of the dark current. (right) Filter mounted above array. The square aperture is part of the light baffle system

The solar calibration aperture provides a means to assess the stability of the system in flight in a manner that is independent of the on-board electronic sources. The input, which is at 90 degrees with respect to the primary aperture, will be pointed at the sun, and the solar radiance will strike a wire mesh system that is near the Optics Box entrance opening (see Fig. 3). The mesh scatters light over a wide enough range of angles that it provides a relatively flat illumination pattern over the entire focal plane after striking both mirrors. (The measured intensity non-uniformity induced by the mesh is $\pm 20 \%$. This effect is accounted for in the calibration.) Since use of the solar calibrator involves pointing the spacecraft, calibration operations that employ it will be done on an occasional basis. The filament and blackbody calibrators will be used to monitor the calibration on a much more frequent basis. The stability of the on-board electronic sources will be monitored using the solar calibrations.

\subsection{The OVIRS Focal Plane}

OVIRS uses linearly variable (also called wedged) filter (LVF) segments to provide spectral differentiation. An LVF is a two-dimensional filter in which the wavelength of transmitted light varies in one dimension, the variable frequency dimension (Rosenberg et al. 1994). The filter segments are bonded into an assembly which is placed directly over the detector. The filters are placed close enough to the optically active surface that each pixel only "sees" light from a section of the filter that is nearly the same size as the pixel. Each pixel along the variable frequency dimension (the row dimension) sees light at a slightly different wavelength than the pixel next to it. However, pixels along the other dimension (the column dimension) all see light of essentially the same wavelength (the variation over the roughly 40 pixels in a column from which data are taken is less than 5\% of the spectral bandwidth). OVIRS uses five LVF segments to provide spectral coverage from 0.4 to $4.3 \mu \mathrm{m}$. This is illustrated in Fig. 5, which shows the Teledyne H1RG array, a schematic of the filter assembly placed over the center of the array, and a picture of the focal plane assembly.

Because the wavelength does not change significantly in the column direction $(<5 \%$ of the FWHM in 40 pixels), several pixels may be summed along a column to reduce the data volume transmitted from the spacecraft. These sums are called "super-pixels" and may be programmed to involve from one to eight pixels, with eight being the number planned for use at Bennu. Bad pixels will be excluded from the sums by using a pre-measured bad pixel map. Enroute to Bennu the bad-pixel map will be checked approximately every six months 
using data taken at the pixel level using both solar calibration data and/or data taken using the on-board calibrators. During operations at Bennu, the bad-pixel map will be checked approximately monthly. The pixels used in the super-pixel sums are taken from the central region of each filter segment and are never closer than 30 rows to the top or bottom of the segment. This minimizes the effect of scattering at the segment boundaries. During prelaunch calibration, measurements were taken that sampled every pixel in the array, including those near the segment boundaries. There was evidence of a small $(<1 \%)$ scattering effect up to $\sim 10$ rows from the boundaries, but by 15 rows, the effect was not measurable. The 30 row exclusion zone was chosen to provide margin on possible scattering. The effect of scattering (if any) will be monitored in flight using the calibration measurements noted above.

The bad pixel-filtered super-pixels received on the ground constitute the Level 0 data. The Level 0 data will be converted to radiance and, to prevent cosmic ray hits or other events from contaminating the spectra, the calibrated data will be processed to flag significant outliers. Currently, the plan is to flag super pixels that are more than 4 standard deviations from the mean of the super pixel column for the current frame, and 2 frames before and 2 frames after it. This process will not catch all events and is only intended as a first step. The flagged, calibrated data will constitute the Level 2 data. Because the rate of wavelength change per pixel in the row dimension is such that two adjacent columns may be summed together without broadening the spectral width, in order to obtain the high SNR required for OVIRS science from the light reflected from a very dark asteroid surface (albedo $\sim 3 \%$ ), higher-level spectral data sets (Level 3 and above) will typically involve summations of super-pixels in both the row and column direction. For example, at Bennu it is planned that four super-pixels will be obtained in each column in each filter segment. Summing two adjacent columns gives a sum of eight super-pixels in a spectral sample. The flagged superpixels will be removed before summing. Thus, up to 64 pixels may be summed into a single spectral sample, increasing the SNR by a factor of 8 above the single-pixel value. Even after the super-pixels are summed into a spectral sample, the slope of the wavelength change is such that there are always at least two spectral samples per resolution element. That is, the spectrum is double sampled.

\section{Electronics}

The OVIRS control electronics consist of three boards; Focal Plane Electronics (FPE), Command and Data Handling (C\&DH), and a Low Voltage Power Supply (LVPS). These are contained within the Main Electronics Box (MEB) mounted directly to the spacecraft on a structural element below the nadir deck (see Fig. 6), and operate at near ambient temperature. The FPE board provides biases and clocks to the focal plane, does a row-by-row reset of the pixels, amplifies the signals from the array, and performs the A/D conversion of the data. It performs these functions using the SIDECAR (System for Image Digitization, Enhancement, Control and Retrieval) ASIC (Application Specific Integrated Circuit) that was developed by Teledyne Imaging Systems (TIS) for use with the HnRG arrays. The science data are converted using 16 bits per pixel. As implemented, the electrical system only adds about $15 \mathrm{e}^{-}$of read noise to the data. This, combined in quadrature with the detector read noise of about $15 \mathrm{e}^{-}$measured for the H1RG detector gives a system read noise (Rn) of $\sim 22 \mathrm{e}^{-}$.

The C\&DH board interprets the commands, does the A/D conversion of the low-speed engineering data, and provides both the high-speed science data interface and the low-speed 

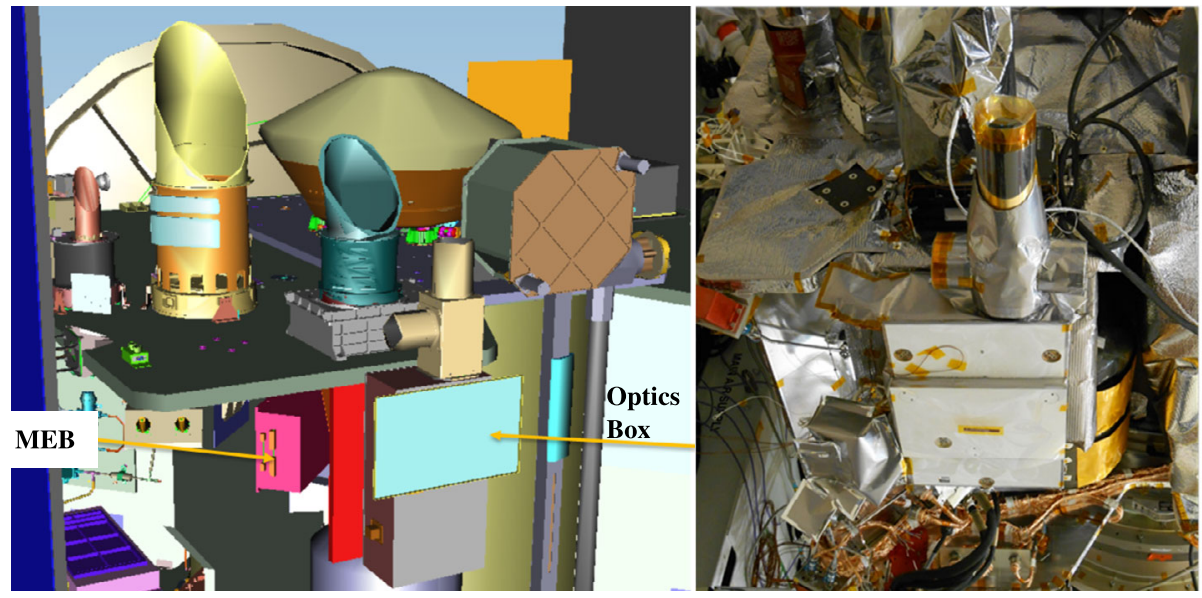

Fig. 6 (left) Drawing showing the spacecraft mounting positions of the MEB and the Optics Box. The Optics Box is mounted on a bracket at the edge of the spacecraft to improve the view of the radiators to deep space. (right) Picture of the OVIRS optics box mounted on the spacecraft

housekeeping data interface. It also performs two critical numerical operations on the data: Correlated Double Sampling (CDS) and the super-pixel summing. During a frame readout, a reset voltage is supplied to all the pixels in each row of the array. The resulting signal at the end of the integration time is relative to this reset level. The reset level of each pixel is read immediately after it is applied. In CDS mode, these reset levels are stored until the integrated signal is read out and then subtracted from the integrated signal to reduce the noise induced by variability in the reset level. Spectral data are usually obtained in CDS mode, although it is possible to return both the reset and the integrated signals. This latter mode, called raw mode, is typically used for anomaly investigations and to check on bias drifts. The C\&DH also performs the super-pixel summing operation. There can be up to eight consecutive pixels in a column in a super-pixel, and up to six super-pixels per column. The minimum integration time is set by how many rows are read out. For bright targets, fewer pixels need to be summed together to get sufficient SNR. In these cases, the number of pixels per super-pixel, and the number of super-pixels can both be reduced. The regions on the array where data is read out (called the Regions of Interest, ROI) can be modified, and all the pixels in the ROI may be read out without doing super-pixel sums. In fact, the entire array may be read out, which is a mode only used for diagnostics. All the options above may be modified in flight by appropriate command and table changes. In the mode that is planned for use at Bennu ( 8 pixels per super-pixel and 4 super-pixels per column) the minimum integration time is 0.303 seconds and it may be increased to 32 times this (9.696 seconds) in steps of 0.303 seconds. The planned integration time at Bennu is 0.909 seconds. Since the predicted noise for a given pixel is: $\left(\mathrm{Rn}^{2}+\right.$ idark $* \mathrm{t}+$ isource $* \mathrm{t}+$ iback $* \mathrm{t})^{1 / 2}$, where $\mathrm{Rn}$ is the system read noise defined above, idark is the dark current, isource is the wavelength dependent signal current produced by the observed object, iback is the wavelength dependent background signal produced by the instrument thermal emission and $t$ is the integration time, it can be seen that the noise contribution for the $200 \mathrm{e}^{-} / \mathrm{s}$ dark current in a $\sim 0.91 \mathrm{~s}$ integration $(\sim 180)$ is more than a factor of 2 less than the $\mathrm{Rn}^{2}$ contribution of $\sim 450$.

The LVPS converts the $30 \mathrm{~V}$ spacecraft power to the various voltages required by OVIRS. The signal produced by the array is sensitive to noise fluctuation, so the voltages output by 


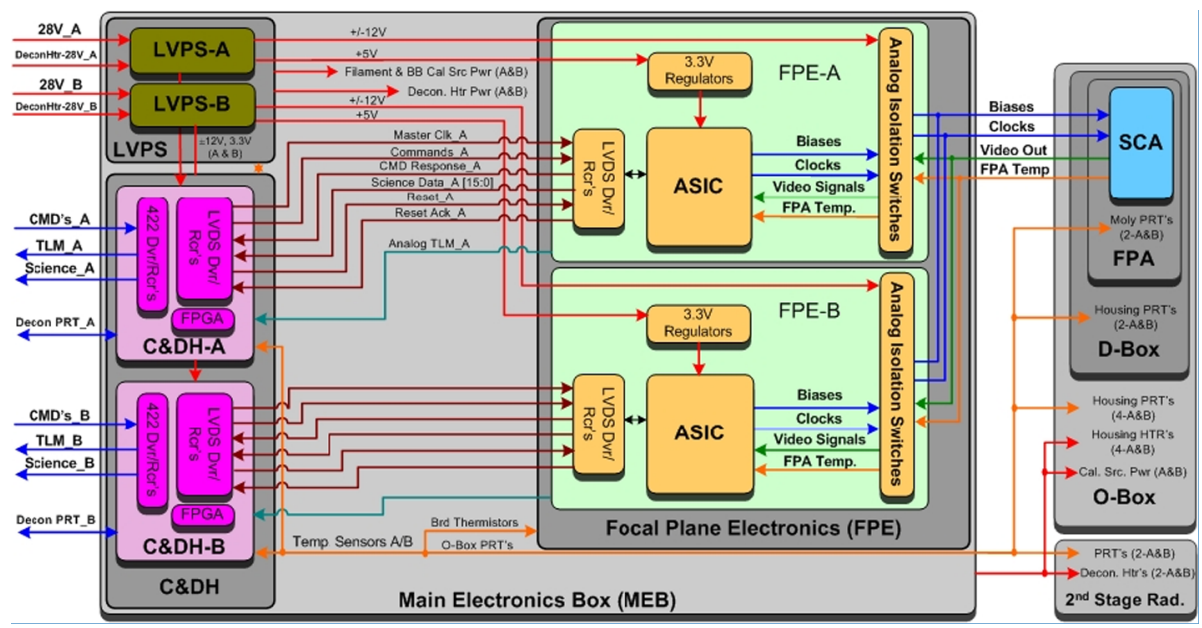

Fig. 7 Block diagram of the MEB showing its interface with both the spacecraft and the Optics Box components. Note that all boards are fully redundant

the LVPS are required to be stable enough that the system read noise is within specifications. The LVPS also supplies the power to the miniaturized blackbody and filament calibrators. The output levels of these devices can be varied such that the maximum temperature the blackbodies can reach is about $\sim 800 \mathrm{~K}$ (but, for lifetime considerations, $\sim 700 \mathrm{~K}$ is the actual temperature used). The maximum temperature of the filaments is $\sim 2300 \mathrm{~K}$. To ensure that the calibration sources are stable, the power supplied to them is controlled. The radiometric outputs and stabilities of the calibrators were established during the instrument-level calibration observations made during thermal vacuum testing. The output levels were determined by comparing the signal observed using the internal calibrations sources at a given setting with the signal observed using the NIST traceable sources. Both internal sources met their $1 \%$ stability requirement, although the blackbody sources did so only after a significant burn-in period of powered operation for about 36 hours. This behavior was expected after ambient storage, and it is planned that the burn-in process will be carried out again soon after launch, after which the sources will remain stable. The total flux level reaching the detectors from the internal calibrators is within an order of magnitude of that produced by Bennu observations, and is much less than 1 milliWatt. This power level does not affect the instrument thermal environment.

OVIRS addresses several of the Level 1 science measurement requirements so the reliability of the electronics is of paramount importance. To ensure that OVIRS is robust, the MEB electronics are fully block redundant with fully isolated A and B sides on each of the electronics boards in the box. Either all the A sides or all of the B sides may be powered on, but both the A sides and the B sides may not be powered on at the same time. The spacecraft also has A and B sides and the spacecraft interfaces are cross-strapped, so that either OVIRS A or B side may be used with either the A or B spacecraft side. This is illustrated in Fig. 7, which shows a schematic of the MEB design. Pictures of the MEB and the electronics boards are shown in Fig. 8. 


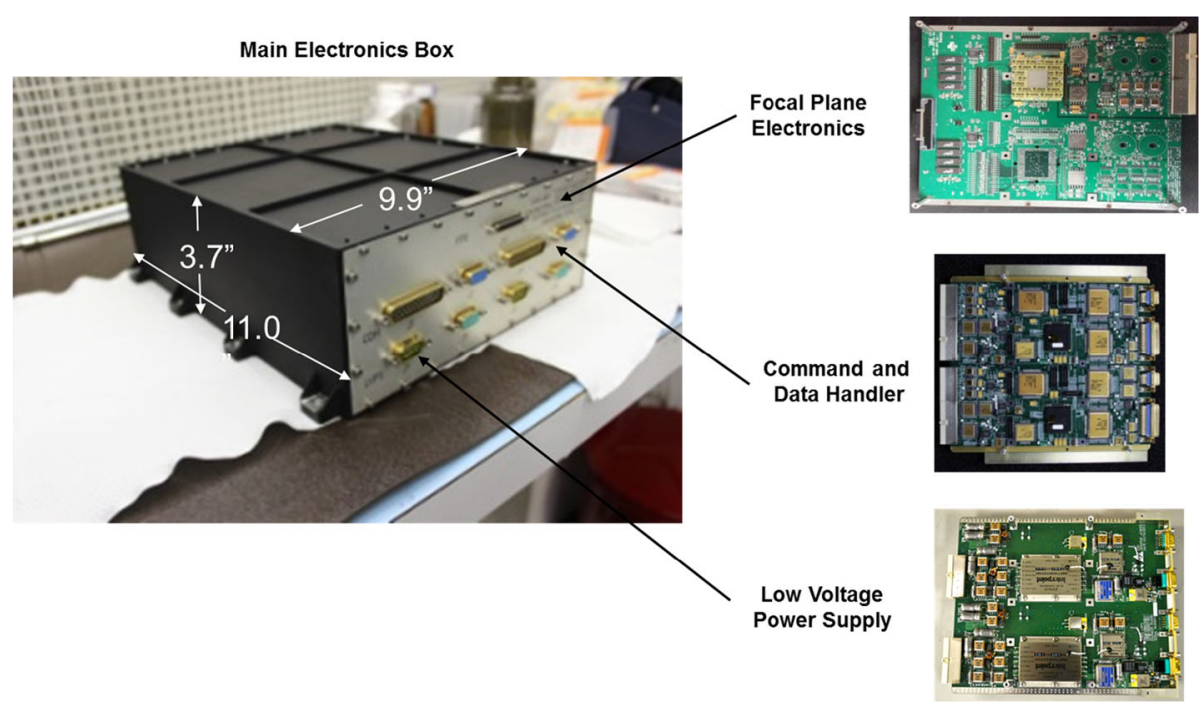

Fig. 8 Pictures of the Main Electronics Box (MEB) and its three cards. The interface connections for each board to both the spacecraft and the Optics Box are also shown (arrows)

\section{Pre-Launch Instrument Characterization}

An extensive pre-launch program of performance verification measurements was carried out for OVIRS at both the component level and the full instrument level, and will be reported elsewhere, though we provide a summary here. The component-level characterization included measurements of the wavelength-dependent quantum efficiency for the array/filter assemblies and measurements of the wavelength dependence of the other optical elements (i.e., reflectance of the mirrors, transmission of the filters, and outputs of the calibration sources). Full instrument-level testing was carried out under spaceflight-like conditions in a thermal vacuum chamber at GSFC. The primary performance characteristics verified in these tests were absolute radiometric calibration of the full system, spectral resolution, spatial profile of the input beam, calibration of the on-board calibration systems, and optical pointing. The directional characteristics of the solar calibration input were also measured. The stability of all these parameters was determined for both instrument sides, and under the thermal variations (detector temperatures of 90-105 K, plus limited tests at higher temperatures of up to $115 \mathrm{~K}$ ) and voltage ranges $(26-30 \mathrm{~V})$ expected to be seen in flight.

The instrument-level tests used a calibration system originally developed for calibrating the Thermal Infrared Sensor (TIRS) instrument on Landsat 8. This system consists of an infrared source module (the IRSM) which illuminates collimating optics through a variety of apertures to simulate targets far removed from the output aperture. The output from the collimation source goes to a steering mirror that projects the beam onto the input of the instrument at a wide range of angles. This allows accurate location of the instrument's boresight. It also allows the off-axis scattering characteristics to be investigated. In addition to the IRSM, there are two NIST-traceable calibration sources in the calibration system, a visible integrating sphere providing visible and near IR radiation and a flood source providing light from the short-wave IR (SWIR) to the Far-IR. The entire calibration system, including the IRSM, the collimation system, the mirrors, the integrating sphere and the flood source, 

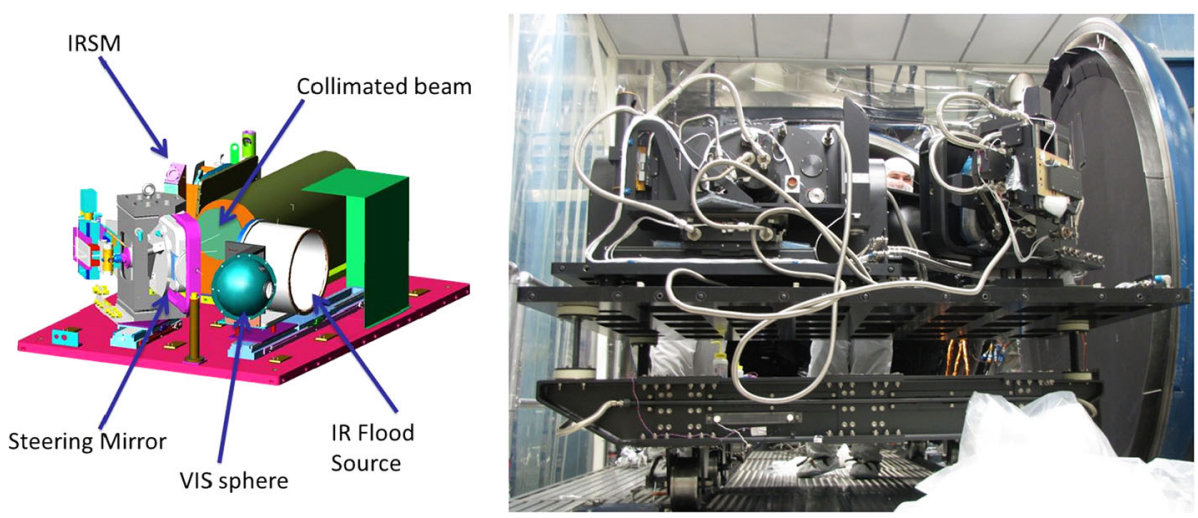

Fig. 9 (a) Schematic of the in-chamber calibration system used to characterize OVIRS performance. (b) The calibration system being readied to enter the chamber

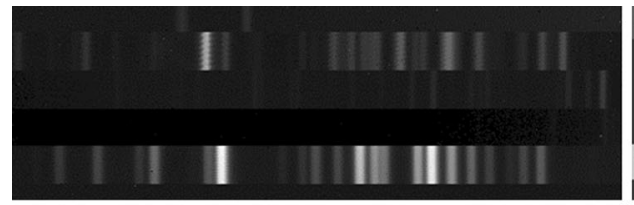

Hg lamp

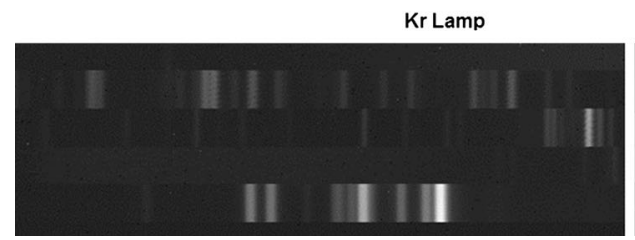

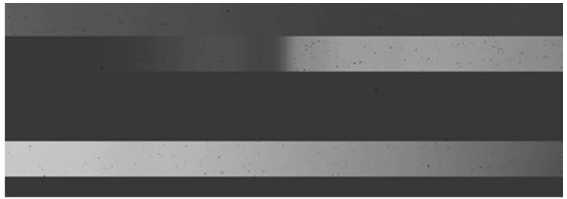

VIS Sphere

IR Flood

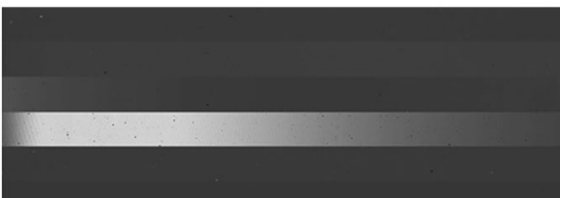

Fig. 10 Spectral output when the instrument is illuminated by sources inside (VIS sphere (top right) and IR Flood (bottom right)) and outside (Kr Lamp (bottom left) and Hg Lamp (top left)) the chamber. The five filter segments are clearly visible, with dark pixel rows at the bottom

goes into the chamber with the instrument. During testing, the components are cooled to near $\mathrm{LN}_{2}$ temperatures so that unwanted thermal signal is minimized. In the chamber, the system may be configured such that light from an outside source can take the place of the IRSM and illuminate the collimating optics. This configuration is used, for example, to introduce the output of a grating monochromator into the system for spectral characterization. For OVIRS, it was also used to allow laser sources and spectral sources, such as Kr lamps, to illuminate the instrument aperture. Figure 9(a) shows a schematic of the calibration system, while Fig. 9(b) shows the system as it is readied to enter the chamber. Figure 10 shows the output observed on the array for various external illumination sources. This figure clearly illustrates the response of individual filter segments when illuminated by sources with different wavelengths. 


\subsection{Measured Parameters}

\subsubsection{Spectral Lineshape and Line Center}

A pixel-by-pixel table of the central wavelength and resolving power was generated for the filter/array focal plane assembly over the entire $0.4-$ to $4.3-\mu \mathrm{m}$ band by using a combination of multi-order grating, laser, and narrow band atomic emission features. In addition, the instrument spectral lineshape (that is, the instrumental response at a given pixel as a function of wavelength) was measured by using multiple grating settings. Figure 11 (top, red line) shows an example of the readout along a single row of one of the LVF segments (the segment called LVF 1b) when the instrument was illuminated using the output of a grating monochromator at a single grating angle setting. In this case the grating input was a visible light source. A blackbody capable of reaching temperatures of $1250 \mathrm{~K}$ was also used for longer wavelengths. The three peaks correspond to orders six through four of the $4-\mu \mathrm{m}$ blazed grating. The top black line shows the readout of the same row when the grating setting is changed by $7.5 \mathrm{~nm}$ in first order (1.5 nm at the $750-\mathrm{nm}$ fifth order). Repeating the operation of changing the grating setting and reading out the rows allows one to characterize the spectral shape of the pixels and is further illustrated in the bottom of Fig. 11, which shows three grating positions for the fifth order. During calibration the grating setting is changed a sufficient number of times that approximately $75 \%$ of the pixels in all rows have their lineshapes sampled from the peak response to $5 \%$ of the peak response. Analysis of these data by fitting the lineshape of each pixel to a Gaussian function allows the central wavelength and the line width (FWHM) of each pixel fit to be determined. This analysis was done for all filter segments, The Gaussian fits reproduced the data to within $10 \%$ of the expected measurement noise indicating that the instrument spectral lineshape is well approximated by a Gaussian function. The estimated error of the pixel line width is $5 \%$ and the error of the line centers is $5 \%$ of the pixel linewidth.

Figure 12 shows the line center/line width results for filter 2, as an example, obtained from an analysis of numerous grating measurements. The left side of the figure shows the wavelength and spectral resolution for a wide range of pixels along a row covered by this filter. The right side shows a plot of the resolving power vs. wavelength resulting from this analysis, showing this filter segment easily meets its requirement.

Fig. 11 (top) Output corresponding to grating orders 6 to 4 . The two lines correspond to two slightly shifted grating positions. (bottom) Three grating positions plotted to show the overlap that is used to determine the lineshape
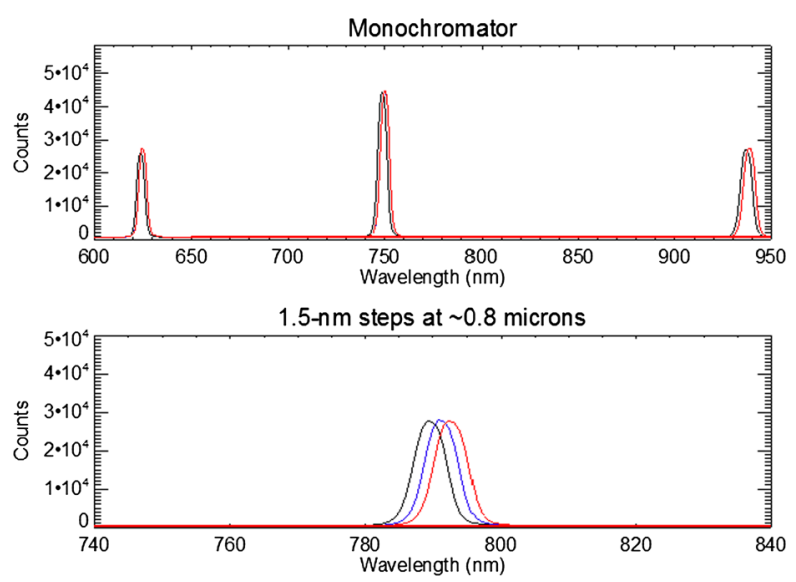

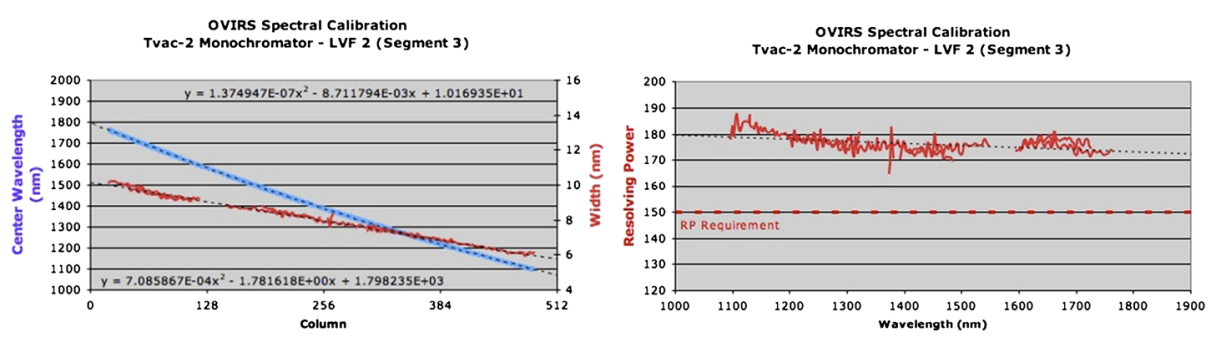

Fig. 12 (left) Plots showing the center wavelength and resolution for the pixels along a row of filter 2 . The top equation is for the resolution, the bottom is for wavelength. (right) Resolving power as a function of wavelength based on the results from the left plot

Fig. 13 Expected OVIRS SNR due to solar reflectance as a function of wavelength for Bennu observations once the asteroid fills the FOV. Assumes a 3\% reflectance and a 1-second integration



\subsubsection{Radiometric Calibration and Sensitivity}

Radiometric calibration is done using the visible/NIR integrating sphere for wavelengths from 400 to $1600 \mathrm{~nm}$ and the IR flood source for wavelengths from about 2500 to 4300 $\mathrm{nm}$. Both sources are calibrated to an accuracy of better than $1 \%$. The calibrations were carried out over a range of temperatures for the flood source (180 to $380 \mathrm{~K}$ ) and over a range of lamp brightnesses for the visible/NIR sphere to check detector linearity and response. Measurements were also carried out at fixed calibration inputs while the MEB input voltages and temperature were varied over the range expected in flight. It was found that the effects of MEB voltage and temperature were negligible. Using visible and IR radiances that correspond to the expected illumination level that would come from Bennu (3-4\% solar reflectance at 1.2 AU from the Sun and $300 \mathrm{~K}$ thermal radiation) and the calibration results, the expected SNR that will be obtained during asteroid operations may be calculated. Figure 13 shows the expected SNR for a 3\% reflective surface when Bennu is $1.2 \mathrm{AU}$ from the sun. Once again, OVIRS exceeds requirements.

\subsubsection{Boresight Pointing and Enclosed Energy}

The OVIRS boresight direction and the enclosed energy as a function of angular spread of the entry beam are determined using a combination of IRSM apertures and the steering mirror. The boresight is best determined by using an aperture near 4 milliradian. The signal 
Table 3 OVIRS driving requirements verification table

\begin{tabular}{|c|c|c|c|c|c|c|}
\hline ID & Requirement & \multicolumn{2}{|c|}{ Required Value } & \multicolumn{3}{|c|}{ Measured Value } \\
\hline OV-3.2 & Overall signal-to-noise ratio & \multicolumn{2}{|l|}{50} & \multicolumn{3}{|l|}{$>50$} \\
\hline OV-3.3 & Accuracy & \multicolumn{2}{|c|}{$\begin{array}{l}2.5 \%(1 \sigma) \text { over a wavelength } \\
\text { span of at least } 0.3 \mu \mathrm{m} \text { within } \\
\text { the spectral region } 0.4 \text { to } 2 \\
\mu \mathrm{m}\end{array}$} & \multicolumn{3}{|c|}{$<0.5 \%$} \\
\hline \multirow[t]{9}{*}{ OV-3.4 } & \multirow{9}{*}{ Encircled energy } & Radius (mrad) & \multicolumn{2}{|l|}{ Requirement } & Measured & Calculated \\
\hline & & 0.5 & \multicolumn{2}{|l|}{-} & $6.7 \%$ & - \\
\hline & & 1.128 & \multicolumn{2}{|l|}{-} & $34.5 \%$ & - \\
\hline & & 2 & \multirow{2}{*}{\multicolumn{2}{|c|}{$83 \%$}} & $95.9 \%$ & - \\
\hline & & \begin{tabular}{|l|l|}
4 \\
\end{tabular} & & $96 \%$ & $97.0 \%$ & - \\
\hline & & 6.735 & \multicolumn{2}{|c|}{$98.5 \%($ at $6 \mathrm{mrad})$} & $98.8 \%$ & - \\
\hline & & 8 & \multicolumn{2}{|c|}{$99.2 \%$} & $99.5 \%$ & - \\
\hline & & 10 & \multicolumn{2}{|l|}{$99.4 \%$} & - & $99.994 \%$ \\
\hline & & $12 *$ & \multicolumn{2}{|l|}{$>99.5 \%$} & $99.8 \%$ & $100 \%$ \\
\hline OV-3.5 & Scattered light & \multicolumn{2}{|c|}{$<0.5 \%$ (12 mrad radius) } & \multicolumn{3}{|c|}{$<0.2 \%$} \\
\hline $\begin{array}{l}\text { OV- } \\
3.6 / 3.7 / 3 . \\
58\end{array}$ & $\begin{array}{l}\text { Instrument Orientation and } \\
\text { Boresight Knowledge }\end{array}$ & \multicolumn{2}{|c|}{$\begin{array}{l}\text { Knowledge to } 0.49 \text { mrad } \\
\text { after post-launch cal } \\
>0.2 \mathrm{mrad} \text { boresight change } \\
\text { in } 1 \mathrm{~s}\end{array}$} & \multicolumn{3}{|c|}{$\begin{array}{l}\text { Max pointing shift } 0.1 \text { to } 0.2 \\
\text { mrad at Op. But once } \\
\text { boresight calibrated cold, } \\
\text { negligible shifts }\end{array}$} \\
\hline OV-3.9 & Instrument Operation & \multicolumn{2}{|c|}{$>5$ continuous hours } & \multicolumn{3}{|c|}{ TVAC $>55 \mathrm{hrs}$} \\
\hline $\begin{array}{l}\text { OV }-3.10- \\
3.13\end{array}$ & Spectral resolution & \multicolumn{2}{|c|}{$\begin{array}{l}\geq 125(.4-1 \mu \mathrm{m}) \\
\geq 150(1-2 \mu \mathrm{m}) \\
\geq 200(2-4.3 \mu \mathrm{m}) \\
\geq 350(2.9-3.6 \mu \mathrm{m})\end{array}$} & \multicolumn{3}{|c|}{$\begin{array}{l}0.4-1 \mu \mathrm{m}:>130 \\
1-2 \mu \mathrm{m}:>170 \\
2-4.3 \mu \mathrm{m}:>225 \\
2.9-3.6 \mu \mathrm{m}:>350\end{array}$} \\
\hline OV-3.36 & Total electrical noise & \multicolumn{2}{|l|}{$35 \mathrm{e}-$} & \multicolumn{3}{|c|}{$20 \mathrm{e}-$} \\
\hline OV-3.48 & Instrument precision & \multicolumn{2}{|c|}{$2 \%(1 \sigma)$ over 5 hours } & \multicolumn{3}{|c|}{$1.9 \%$} \\
\hline
\end{tabular}

produced by the array is maximized when the collimated beam is directly along the boresight, and decreases when it is pointed off it. The pointing was found to be the same for apertures smaller than 4 milliradian as well. Once the boresight was determined, the angular dependence of enclosed energy was determined by pointing collimated beams corresponding to angular spreads of $25 \%$ of the 4 -milliradian FOV to six times the FOV. The results of these measurements, as well as those for all the driving requirements verified during the thermal vacuum testing calibration effort, are summarized in Table 3. In all cases OVIRS meets or exceeds its required performance.

\section{Planned In-Flight Instrument Calibration}

OVIRS calibration will continue in flight. The OSIRIS-REx launch occurred on September 9, 2016. Roughly two weeks after launch, the instrument was activated for a series of instrument functional checkouts. This was done with the decontamination heaters on, so science performance could not be assessed. The instrument functioned nominally. The voltages reported by the spacecraft housekeeping telemetry were consistent with expectations. The instrument responded properly to commands and the science data returned, though saturated, were consistent with pre-launch measurements.

On October 10, the decontamination heaters were turned off for about 82 hours. The detector cooled to about $106 \mathrm{~K}$, and the temperatures measured by sensors located at several places on the optics box ranged from 153 to $155 \mathrm{~K}$. This allowed the acquisition of dark 


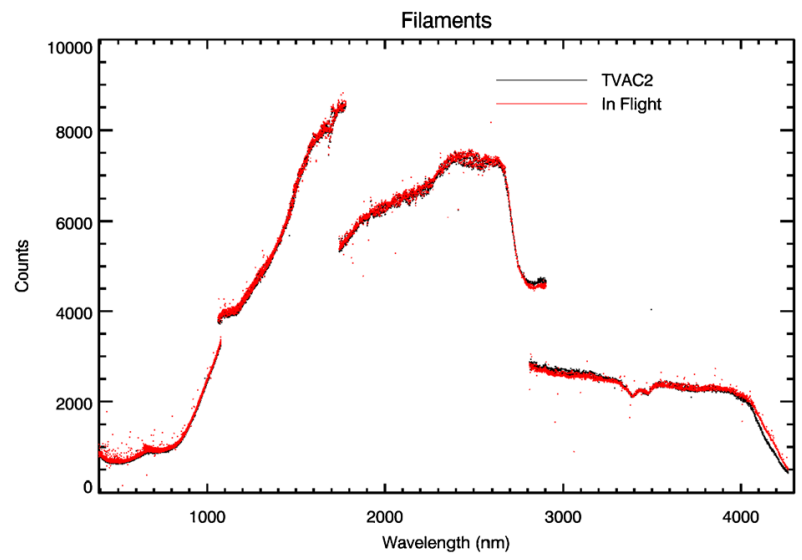

Fig. 14 Comparison between the pre-launch counts obtained from the filament calibrator in a 1-second integration (black dots) with those obtained at the same power setting about 1 month after launch (red dots). For the required 1- to 4-micron spectral range, the data overlap at the level of the noise. These are data for every pixel in the ROI; super-pixel summing is not done. At wavelengths $>4$ microns, the apparent flight flux is slightly higher than the pre-launch flux, but that is probably due to an incorrect background correction in the pre-launch data. Note the spectral feature at about 3.5 microns. This is caused by an absorption feature in the filament bulb and indicates that there has been no wavelength shift post-launch, at least in segment 4

sky data and filament source calibrations for comparison with pre-launch measurements. Figure 14 shows a comparison between the filament measurements pre-(white dots) and post-launch (red dots). These data are taken in CDS mode, and all pixels within the ROIs were downlinked (there was no super-pixel summation). All pixels, including bad pixels, are plotted. As may be seen from the figure, for the good pixels, the pre- and post-launch data overlap at the level of the expected single-pixel error (50 to 100 counts).

A solar calibration is planned for early February which will include a set of slews to determine the optimal pointing for the solar calibrator. This will allow the solar calibration results to be compared with pre-launch measurements of the filament and miniaturized blackbody sources. Following this, deep space and on-board calibration source measurements will occur on an occasional basis. During the Earth gravity assist that will occur in September of 2017 a fairly active calibration campaign will be carried out using both the Earth and the Moon as calibration targets. This will not only provide an opportunity to verify the radiometric calibration, but molecular features in Earth's atmosphere will also allow us to verify the spectral calibration post-launch. The Earth flyby will also serve as a valuable practice opportunity for Bennu operations.

Afterward, during the remaining cruise phase to Bennu, there will be occasional calibrations made using the filaments and the blackbodies, and two solar calibrations are also planned. At Bennu, the frequency of calibration source measurements will increase. In fact, it is expected that calibrations will be performed before and after asteroid scans and possibly even within the scans themselves. The solar calibration cadence will also increase.

\section{Post-Calibration Data Processing}

As discussed in Sect. 3, a raw OVIRS spectrum consists of data from each of the five filter segments summed into super-pixels. The Level 2 data are calibrated, with bad pixels flagged. 
Thus, each super-pixel is assigned a wavelength and a calibrated radiance. Because of the filter design, there is some spectral overlap from segment to segment and in the Level 2 archived data (see Table 2). These data will be made available to the Planetary Data System. For use in science analysis, the spectra will be further processed by the OSIRIS-REx science team into Level 3 products. First, the spectrum will be resampled to remove the spectral overlap, pixels of similar wavelength will be averaged together, and each spectrum will be placed onto a consistent wavelength grid, with 2-nm spacing for wavelengths $<2.4$ microns and 5-nm spacing at longer wavelengths. The next step in data processing is thermal tail removal, performed by using the surface temperature obtained from OTES data acquired with the same geometry (and preferably near simultaneous) to the OVIRS data, to remove the thermal effect from the OVIRS spectrum. Lastly, the team will convert the spectrum to I/F. Here, the OVIRS spectrum is divided by a Project-defined solar spectrum, rangecorrected to the solar flux at Bennu. These Level 3 spectra, with their associated wavelengths and uncertainties, will also be available to users in the Planetary Data System.

\section{Conclusion}

This paper describes the design, operation, and performance of OVIRS, a highly capable remote sensing visible/SWIR spectral imager flying on the OSIRIS-REx mission to the nearEarth carbonaceous asteroid, Bennu. OVIRS consists of a set of collimating optics feeding a focal plane, whose spectral response covers the $0.4-$ to $4.3-\mu \mathrm{m}$ spectral band. OVIRS will obtain spectral maps of the entire surface of Bennu at a spatial resolution of 20 meters or better. It will also obtain spectral maps with spatial resolution of 2 meters or higher of areas that are potential sites for the spacecraft to collect a sample that will be returned to Earth. The spectral data will be used to identify the minerals, chemicals, and molecular species on the surface including, most importantly, organic species. These data will help guide the process of sample site selection; they will provide a connection between the geology of the surface and the compositions determined from laboratory investigations of the sample when it is back on Earth; they will provide clues to the space weathering of the surface; and they will be part of the analysis of the Yarkovsky effect, which can change the orbital characteristics of asteroids, causing them to become hazards.

OVIRS has been extensively tested at the component and full-instrument level. These tests have verified that it meets all its performance requirements with margin. OVIRS will provide a wealth of information on the composition, morphology, and thermal characteristics of Bennu. The data it produces during its nearly year-long period of active observations promise to significantly advance our understanding of this small body and others like it. In combination with the information obtained by the other instruments on the mission, and the OTES thermal spectrometer in particular, it will shed new light on the evolution of our solar system and the nature of the objects that may have brought the building blocks of life to our planet.

Acknowledgements The authors would like to thank the entire OVIRS support teams at GSFC, SwRI, and $\mathrm{J} \& \mathrm{~T}$ for their untiring efforts in making OVIRS a reality. The contributions of JDSU/Uniphase, Teledyne, and Corning Diamond Turning Division are also gratefully acknowledged. The authors would also like to thank the PI office at the University of Arizona, the Project Office at GSFC, the New Frontiers Program Office at MSFC, and NASA HQ for their support.

Open Access This article is distributed under the terms of the Creative Commons Attribution 4.0 International License (http://creativecommons.org/licenses/by/4.0/), which permits unrestricted use, distribution, and reproduction in any medium, provided you give appropriate credit to the original author(s) and the source, provide a link to the Creative Commons license, and indicate if changes were made. 


\section{References}

H. Campins, K. Hargrove, N. Pinilla-Alonso, E.S. Howell, M.S. Kelley, J. Licandro, T. Mothé-Diniz, Y. Fernández, J. Ziffer, Water ice and organics on the surface of the asteroid 24 Themis. Nature 464, 13201321 (2010)

M.C. De Sanctis, E. Ammannito, H.Y. McSween, A. Raponi, S. Marchi, F. Capaccioni, M.T. Capria, F.G. Carrozzo, M. Ciarniello, S. Fonte, M. Formisano, A. Frigeri, M. Giardino, A. Longobardo, G. Magni, L.A. McFadden, E. Palomba, C.M. Pieters, F. Tosi, F. Zambon, C.A. Raymond, C.T. Russell, Localized aliphatic organic material on the surface of Ceres. Science 355, 719-722 (2017)

M.J. Gaffey, Space weathering and the interpretation of asteroid reflectance spectra. Icarus 209, 564-574 (2010)

T. Hiroi, M.E. Zolensky, C.M. Pieters, The Tagish Lake meteorite: a possible sample from a D-type asteroid. Science 293, 2234-2236 (2001)

D.S. Lauretta, A.E. Bartels, M.A. Barucci, E.B. Bierhaus, R.P. Binzel, W.F. Bottke, H. Campins, S.R. Chesley, B.C. Clark, B.E. Clark, E.A. Cloutis, H.C. Connolly, M.K. Crombie, M. Delbó, J.P. Dworkin, J.P. Emery, D.P. Glavin, V.E. Hamilton, C.W. Hergenrother, C.L. Johnson, L.P. Keller, P. Michel, M.C. Nolan, S.A. Sandford, D.J. Scheeres, A.A. Simon, B.M. Sutter, D. Vokrouhlický, K.J. Walsh, The OSIRIS-REx target asteroid 101955 Bennu: constraints on its physical, geological, and dynamical nature from astronomical observations. Meteorit. Planet. Sci. 50, 834-849 (2015)

D.S. Lauretta, S.S. Balram-Knutson, E. Beshore, W.V. Boynton, C. Drouet d'Aubigny, D.N. DellaGiustina, H.L. Enos, D.R. Gholish, C.W. Hergenrother, E.S. Howell, C.A. Johnson, E.T. Morton, M.C. Nolan, B. Rizk, H.L. Roper, A.E. Bartels, B.J. Bos, J.P. Dworkin, D.E. Highsmith, M.C. Moreau, D.A. Lorenz, L.F. Lim, R. Mink, J.A. Nuth, D.C. Reuter, A.A. Simon, E.B. Bierhaus, B.H. Bryan, R. Ballouz, O.S. Barnouin, R.P. Binzel, W.F. Bottke, V.E. Hamilton, K.J. Walsh, S.R. Chesley, P.R. Christensen, B.E. Clark, H.C. Connolly, M.K. Crombie, M.G. Daly, J.P. Emery, T.J. McCoy, J.W. McMahon, D.J. Scheeres, S. Messenger, K. Nakamura-Messenger, K. Righter, S.A. Sandford, OSIRIS-REx: sample return from asteroid (101955) Bennu. Space Sci. Rev. (2017). https://doi.org/10.1007/s11214-017-0405-1 (this volume)

J. Licandro, H. Campins, M. Kelley, K. Hargrove, N. Pinilla-Alonso, D. Cruikshank, A.S. Rivkin, J. Emery, (65) Cybele: detection of small silicate grains, water-ice, and organics. Astronomy \& Astrophysics 525, A3 (2011)

D.C. Reuter, S.A. Stern, J. Scherrer, D.E. Jennings, J. Baer, J. Hanley, L. Hardaway, A. Lunsford, S. McMuldroch, J. Moore, C. Olkin, R. Parizek, H. Reitsma, D. Sabatke, J. Spencer, J. Stone, H. Throop, J. Van Cleve, G.E. Weigle, L.A. Young Ralph, A visible/infrared imager for the New Horizons Pluto/Kuiper Belt mission. Space Sci. Rev. 140, 129-154 (2008)

A.S. Rivkin, J.P. Emery, Detection of ice and organics on an asteroidal surface. Nature 464, 1322-1323 (2010)

K.P. Rosenberg, K.D. Hendrix, D.E. Jennings, D.C. Reuter, M.D. Jhabvala, A.T. La, Logarithmically variable infrared etalon filters, in Optical Thin Films IV: New Developments, San Diego, CA, 25-27 July, 1994. SPIE Proceedings, vol. 2262 (1994)

D. Vokrouhlicky, A. Milani, S.R. Chesley, Yarkovsky effect on small near-Earth asteroids: mathematical formulation and examples. Icarus 148, 118-138 (2000) 